\title{
In Search of an Explanatory Bridge: Where Oriental and Western Medicine Converges
}

\author{
Jessica Dicker* \\ Southwest Acupuncture College, USA
}

*Corresponding author: Jessica Dicker, Southwest Acupuncture College, USA

Received Date: November 09, 2019

Published Date: November 22, 2019

\begin{abstract}
Oriental Medicine uses metaphoric language to express concepts that apply universally. Often, it uses the concepts of energy and describes things at an energetic level. Conversely, Western Medicine uses concepts of chemistry, atoms and molecules, where descriptors are at the micro-level by way of reductionism. The former sounds rather esoteric, while the latter tends to portray a modern scientific view. It is often said that one cannot compare Oriental Medicine theories with that of Western Medicine. However, this assertion may not be necessary. Although there are still mysteries yet to be unfolded, Oriental Medicine can be explained with scientific notions. If one can translate the language spoken by each of the two medicine traditions, one may find a bridge where the two medicines resonant beautifully.
\end{abstract}

Keywords: Oriental medicine; Western medicine; Chemistry; Energy; Meridian

\section{Introduction}

Gazing at a map depiction of the human body circulatory system, one can easily imagine the system as a chemical plant by which a vast amount and variety of chemical compounds are constantly being processed. There are lengthy pipes or vessels, complex portals, hydrants, numerous valves, pools, filters, and outlets which ensure that there is correct flow, appropriate product generation, and of course, contingency or emergency operations. Any interrupted flow within the pipes due to leaking valves or clotted portal, filters, etc. can cause a systematic problem - it is called a disease in Western medical terms, and a disharmony in Oriental medical language. The approach to problem resolution may differ, but they share some fundamental underlying elements that unifies these two medical traditions and forms the basis by which we can begin to bridge the language barrier.

\section{Toxin elimination through blood letting}

One of the most significant documents on Oriental Medicine theory is the "Yellow Emperor's Classic of Internal Medicine." In this series, it is recorded that "a disease is to be removed quickly by draining the evil (pathogen) and letting the blood" [1]. Western medical physicians, likewise, have historically used bleeding techniques to intentionally liberate the infected blood or toxin from inside the body. Although actual bleeding is rarely seen in today's biomedical practice, medicinal leech therapy is still a common and valid form of treatment. Leech suction provides a conduit for the body to discharge its burden of toxin [2-4]. The classic of Chinese medicine text claims that the body has pathways that are designed to hold pathogens at bay as to protect essential organs $[1,5]$. These pathways are part of the body's defense system - a special part of the meridian system. However, the capacity of this system is limited - when it is filled and overflows, the frontline of the battle is lost. A scenario for a disharmony in a meridian would be like this -something is lodged in a pipe, the closest valve needs to be opened, and the obstacle extracted, to resolve the problem. In the same sense, if there is an outlet and the intruding pathogen is diverted to outside the body, rather than to allow further invasion, it is therapeutic to the body. This protocol is done via bleeding the Luo meridian (collaterals). The Luo-connecting point of each meridian is the special valve which provides the emergent outlet. In fact, most of the Luo-connecting points of the twelve meridians are located slightly off the main trajectory, indicating an outlet energetic.

\section{Emotion and the Liver Connection}

Effects due to emotion is another example where we can see a connection between the two traditions. Emotions arise from 
various elements; one important mechanistic component in Western medicine is neurotransmitters. Brain chemistry does not directly cause emotions but is essentially involved and shapes our emotional responses and experiences. As emotion arise, the feelings of good or bad, high or low are manifestations of neurotransmitters, hormones, enzymes, etc. rapidly transmitting, transferring, and transforming from organ to organ, system to system. Emotions are abstract and subjective, but corresponding chemistry inside the body is substantial and objective. Other than the physiological relationship of emotions with limbic systems, biologically, most neurotransmitters, once released into the blood stream, are removed or processed by the liver, except acetylcholine and some peptides which depend on enzymes for removal from the synaptic clefts [6].

Oriental medicine almost exclusively contributes emotional disorder to liver disharmony, well before there was any understanding of chemical homeostasis occurring along with the emotional ups and downs. It is not hard to understand that the liver's functionalities can be off balance due to excessive chemical processing load associated with emotions, particularly when there is extreme fear, anger, anxious, or other so-called emotional disorders.

\section{The migraine and the liver connection}

The migraine is a good case in point where biochemical effects and liver pathology in Oriental medicine are related. The cause of migraine remains unclear within Western medicine circles. However, research has convincingly shown that biochemically, systemic serotonin (5-HT) and its metabolite 5-hydroxyindoleacetic acid (5-HIAA) are involved [7,8]. Within brain chemistry, serotonin is known to interconnect mood, behavior, learning, sleep and anxiety to name but a few. Peripherally, serotonin highlights sensitivity of the pain pathway serotonin vasoconstricts the nerve endings and blood vessels, thus affecting nociceptive pain. Studies have noted that migraineurs had lower plasma 5-HT and higher 5-HIAA levels between attacks [8]. Urinary excretion of 5-HIAA also increased during an attack and then return to normal afterward $[9,10]$. Additionally, research has concluded that 5-HT is involved in liver biology and physiology, such as regulate several key aspects of liver biology and these functions include hepatic blood flow, innervation and tissue regeneration. RG Ruddell \& PA Lang [11,12] While biomedicine considers serotonin is a major player to cause migraine, Oriental medicine attribute migraine as the liver disharmony. The relationship between serotonin and the liver provides a sound evidence for the convergence of the two medicines.

Common triggers of migraine are as follow [13]: hormonal change, stress, lack of sleep, foods, additives, alcohol, caffeine, and medication overdose, all of which are indicative of chemical changes inside the body, presumably and consequently, the liver is overburdened with chemical processing. On the other hand, the majority of serotonin (90\%) is synthesized by enterochromaffin cells of the gastrointestinal tract and from there it is exported to various sites around the body [11]. Reasonably, when the liver is distressed, the functionality of the gastrointestinal system is inhibited, causing a reduction in serotonin synthesis. When the serotonin level drops during a migraine, the hepatic blood flow is decreased, feasibly due to the vasoconstriction effect. In other words, the biological and chemical level changes inside the liver play a huge role in the migraine. This helps construct a bridge between Western and Oriental medical explanation for the migraine- the main disease mechanism of migraine headaches involves the liver at its energetic level - the liver is in disharmony [14].

Oriental Medicine depicts the liver meridian trajectory as going from the hallux to the top of the head, interconnecting the liver, stomach, lungs and eyes. According to Oriental medicine, the liver is one of the three main mediators to the blood, particularly, blood flow. When blood flow in the liver is constricted, the flow of Qi, like a weak electrical current along the fascia D Keown [15], is constrained as well. When the smooth flow of Qi is obstructed on its way to the head, headaches occur. If it is hindered to the stomach, vomiting or nausea occurs in conjunction with head pain. Likewise, various eye issues may occur with the onset of a debilitating headache due to disharmony and disturbance in the liver energetic trajectory. While Oriental medicine theory tries to explain migraines in an energetic way, it is often coherent with current biomedicine understanding of migraine within a biological and biochemical model.

\section{Conclusion}

Oriental medicine provides a "big picture" explanation of the body, while Western medicine tends to focus more at the atomic or molecular level to understand the mechanics. Even though these two medical traditions use completely different languages, they are in many ways, complementary Oriental medicine speaks of energy, while Western medicine atoms and molecules, together they reflect an incredible intellectual design energy is stored in atoms and molecules.

\section{Acknowledgement}

Dr Katelijne Flies, Department of Biology and Biotechnology, Central New Mexico (CNM) Community College, for her valuable insightand recommendations on biochemistry of neurotransmitters.

\section{Conflicts of Interest}

No conflict of interest.

\section{References}

1. PU Unschuld (2016) Yellow Emperor's Classic of Internal Medicine-Ling Shu. The Ancient Classic on Needle Therapy. The Complete Chinese Text with an Annotated English Translation, University of California Press, USA.

2. AK Sig, M Guney, AU Guclu, E Ozmen (2017) Medicinal Leech Therapy-an overall perspective. Integr Med Res 6(4): 337-343.

3. Michalsen A, S Klotz, R Luedtke, S Moebus, G Spahn, et. al. (2003) Effectiveness of leech therapy in osteroarthritis of the knee: a randomized, controlled trial. Ann Intern Med 139(9): 724-730.

4. AP Singh (2010) Medicinal leech therapy (Hirudotherapy): A brief overview. Complement Ther Clin Pract 16(4): 213-215. 
5. Cecil Sternman (2018) Advanced Acupuncture- A Clinic Manual, Protocols for the Complement Channels of the Complete Acupuncture System: The Sinew, Luo, Divergent and Eight Extraordinary Channels. ( $2^{\text {nd }}$ edn), Classical Wellness Press, New York, USA.

6. D Purves, GJ Augustine, D Fitzpatrick (2001) Neuroscience (2 ${ }^{\text {nd }}$ edn) Sunderland (MA): Sinauer Associates. Neurotransmitter Release and Removal.

7. E Hamel (2007) Serotonin and migraine: biology and clinical implications. Cephalalgia 27(11): 1293-1300.

8. SD Silberstei (1994) Serotonin (5-HT) and migraine. Headache 34(7): 408-417.

9. MD Ferrari, J Odink, C Tapparelli, GMJ Van Kempen, EJM Pennings, et al. (1989) Serotonin metabolism in migraine. Neurology 39(9): 1239-1242.
10. M Larkin, The Role of Serotonin in Migraine, JAMA Migraine Information.

11. RG Ruddell, DA Mann, GA Ramm (2008) The function of serotonin within the liver. J Hetapology 48(4): 666-675.

12. PA Lang, C Contaldo, P Georgiew, AM El Badry, M Recher, et al. (2008) Aggravation of viral hepatitis by platelet-derived serotonin. Nat Med 14(7): 756-761.

13. Migraine, Symptoms and Causes, Mayo Clinic, USA

14. B Flaws, P Sionneau (2005) The Treatment of Modern Western Medical Disease with Chinese Medicine- A Textbook and Clinical Manual, $\left(2^{\text {nd }}\right.$ edn), Blue Poppy Press, CO, USA, pp. 365.

15. D Keown (2014) The Spark in the Machine: How the Science of Acupuncture Explains the Mysteries of Western Medicine. $\left(1^{\text {st }}\right.$ edn), Singing Dragon, London, United Kingdom. 